\title{
Convexity limit angles for isoptics
}

\section{Magdalena Skrzypiec ${ }^{1}$}

Received: 8 May 2020 / Accepted: 1 February 2021 / Published online: 4 March 2021

(C) The Author(s) 2021

\begin{abstract}
Given an oval $C$ in the plane, the $\alpha$-isoptic $C_{\alpha}$ of $C$ is the plane curve composed of the points from which $C$ can be seen under the angle $\pi-\alpha$. We consider isoptics of ovals parametrized with the support function $p(t)=a+\cos n t, n \in \mathbb{N}$, and present an example of an oval such that when $\alpha$ increases, the $\alpha$-isoptics begin to be convex, then lose their convexity and finally are convex again along a curve intersecting the isoptics orthogonally. Next we give an example of a curve from the same family, for which the curvature of the isoptics changes its sign three times. These changes occur on the symmetry axes of the oval $C$ and coincide with the orthogonal trajectories which start at the points with extremal curvature. Finally, we formulate the hypothesis concerning the general case where we expect $n-1$ convexity limit angles for the isoptics of an oval parametrized by $p(t)=a+\cos n t$.
\end{abstract}

Keywords Isoptic curve $\cdot$ Convex curve $\cdot$ Limit angle $\cdot$ Support function

Mathematics Subject Classification 53A04 $\cdot 53 \mathrm{C} 44 \cdot 53 \mathrm{~A} 25$

\section{Introduction}

Isoptic curves were defined in 1704 by Philipe de La Hire, as mentioned in Lawrence (1972) p. 58. These curves remain interesting, as can be seen for example in Kunkli et al. (2013) and Dana-Picard (2020). Isoptics also have applications in mechanics and optics (see Weiss and Martini 2000; Wunderlich 1971).

Isoptics of convex curves are not necessarily convex. For isoptics of an ellipse, in Miernowski and Mozgawa (1997) was introduced the notion of the limit angle, described below.

Magdalena Skrzypiec

mskrzypiec@ hektor.umcs.lublin.pl

1 Institute of Mathematics, Maria Curie-Sklodowska University, pl. M. Curie-Skłodowskiej 1, 20-031 Lublin, Poland 
The inspiration to study this problem came about when we noticed certain irregularities. These were on graphs of the dual curves to isoptics of the oval, with support function $p(t)=10+\cos 3 t$, see Skrzypiec (2018).

The results presented below have application to optics and architecture (i.e., lighting and shades).

\section{Isoptics with two limit angles}

Theorem 1 Fix $8<a<8 \sqrt{2}$ and let $C$ be the oval described by the support function $p(t)=a+\cos 3 t$. For $\alpha \in(0, \pi)$, let $C_{\alpha}$ be the $\alpha$-isoptic of $C$. Then there exist angles $0<\alpha_{1}<\alpha_{2}<\pi$ such that the point of $C_{\alpha}$ which intersects the positive $X$-axis has a positive curvature if $\alpha \in\left(0, \alpha_{1}\right) \cup\left(\alpha_{2}, \pi\right)$ and a negative curvature if $\alpha \in\left(\alpha_{1}, \alpha_{2}\right)$.

Remark 1 An angle $\alpha_{0}$ for which $C_{\alpha}$ is convex for $\alpha<\alpha_{0}$ and concave for $\alpha_{0}<\alpha$ ( $\alpha$ near $\alpha_{0}$ ) is called a limit angle (Miernowski and Mozgawa 1997). For the sake of clarity and generality, we call it a convexity limit angle, and extend its use also in the case where the sign of the curvature is considered only along a curve intersecting the isoptic orthogonally.

Proof Using the parametrization

$$
z_{\alpha}(t)=p(t) e^{i t}+\left\{-p(t) \cot \alpha+\frac{1}{\sin \alpha} p(t+\alpha)\right\} i e^{i t}, t \in \mathbb{R},
$$

of isoptics from Cieślak et al. (1991), we prove that the curve $C$ given by

$$
z(t)=p(t) e^{i t}+p^{\prime}(t) i e^{i t} \text { for } t \in[0,2 \pi),
$$

with the support function $p(t)=a+\cos 3 t$, where $a>8$ for some values of $a$, is the example needed in our theorem.

Let us notice that this curve has three axes of symmetry. One of them is the $X$ axis. We obtain the others by rotations by the angles $\frac{2}{3} \pi$ and $\frac{4}{3} \pi$. Each of these axes of symmetry passes through points of extremal curvature of $C$. Isoptics $C_{\alpha}$ are symmetric with respect to the same axes of symmetry as $C$. That is why it is enough to study them in the neighbourhood of one of these symmetry axes. Without loss of generality, we choose the $X$-axis.

Let us recall that the curve, which in each point forms a right angle with a curve from a given family of curves, is called the orthogonal trajectory. It is easy to see that orthogonal trajectories of isoptics of the curve with the support function $p(t)=$ $a+\cos 3 t$, starting with the points of greatest and smallest curvature, coincide with the parts of the symmetry axes mentioned above. Using the parametrization (1) of isoptics, a straightforward computation shows that the points $z_{\alpha}\left(-\frac{\alpha}{2}\right)$ lie on the positive $X$-axis. Tangent vectors of isoptics at these points are equal to

$$
z_{\alpha}^{\prime}\left(-\frac{\alpha}{2}\right)=i \frac{2\left(p\left(\frac{\alpha}{2}\right) \sin \frac{\alpha}{2}-p^{\prime}\left(\frac{\alpha}{2}\right) \sin \frac{\alpha}{2}\right)}{\sin \alpha} .
$$



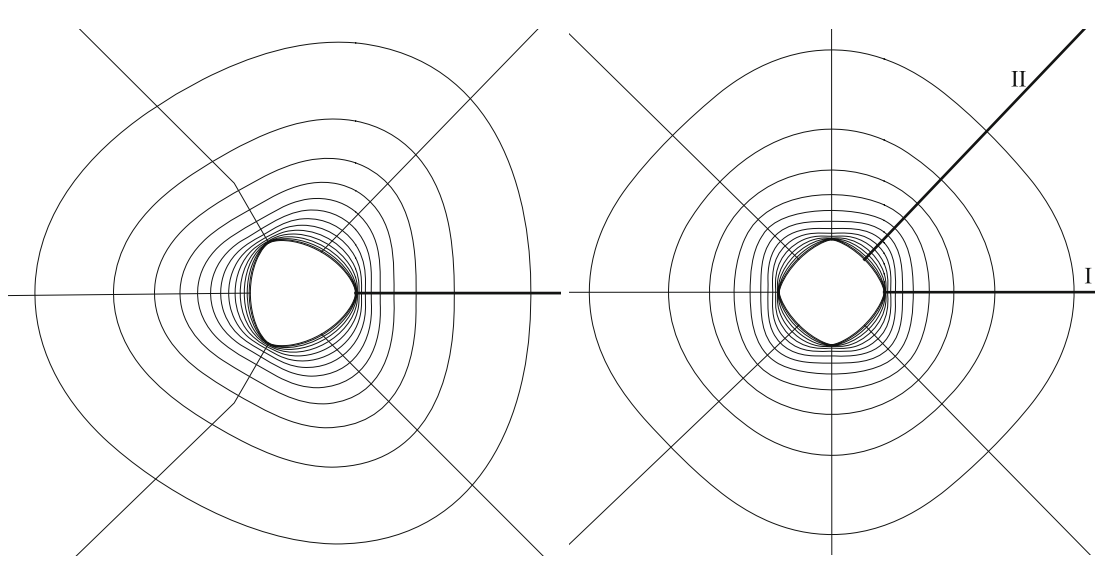

Fig. 1 Isoptics of curves with the support function $p(t)=10+\cos 3 t$ (left) and $p(t)=16+\cos 4 t$ (right), along with some of their orthogonal trajectories

Note that they are vertical. Therefore the set of points $\left\{z_{\alpha}\left(-\frac{\alpha}{2}\right), \alpha \in(0, \pi)\right\}$ forms the orthogonal trajectory of isoptics, starting at $z(0)$. Similarly, the points $z_{\alpha}\left(\pi-\frac{\alpha}{2}\right)$ lying on the negative $X$-axis form the orthogonal trajectory, starting at $z(\pi)$. The first derivative of the curvature of isoptics (see Cieślak et al. 1991)

$$
k_{\alpha}(t)=\frac{\left[z_{\alpha}^{\prime}(t), z_{\alpha}^{\prime \prime}(t)\right]}{\left|z_{\alpha}^{\prime}(t)\right|^{3}}=\frac{\sin \alpha}{|q(t)|^{3}}\left(2|q(t)|-\left[q(t), q^{\prime}(t)\right]\right)
$$

is zero along these trajectories.

We now compute the number of sign changes of the curvature of isoptics, along these trajectories. Let us consider the curvature of the isoptic of the curve with support function $p(t)=a+\cos 3 t$. It is given by the formula

$$
k_{\alpha}(t)=\frac{n_{\alpha}(t)}{d_{\alpha}(t)},
$$

where

$$
\begin{aligned}
n_{\alpha}(t)= & 24+a^{2}+20 \cos \alpha-8 \cos 2 \alpha-4 \cos 3 \alpha+a \cos 3 t+a \cos 3(t+\alpha) \\
& +8 \cos 3(2 t+\alpha)-9 a \cos (3 t+\alpha)+8 \cos 2(3 t+\alpha)-9 a \cos (3 t+2 \alpha) \\
& +4 \cos (6 t+\alpha)+8 \cos (6 t+4 \alpha)+4 \cos (6 t+5 \alpha)
\end{aligned}
$$

and

$$
\begin{aligned}
d_{\alpha}(t)= & \frac{1}{\cos \frac{\alpha}{2}}\left(12+a^{2}+14 \cos \alpha+4 \cos 2 \alpha+2 \cos 3 \alpha-2 a \cos 3 t-2 a \cos 3(t+\alpha)\right. \\
& +8 \cos 3(2 t+\alpha)-6 a \cos (3 t+\alpha)+8 \cos 2(3 t+\alpha)-6 a \cos (3 t+2 \alpha) \\
& +4 \cos (6 t+\alpha)+8 \cos (6 t+4 \alpha)+4 \cos (6 t+5 \alpha)) .
\end{aligned}
$$


We notice that the denominator of this curvature is always positive since the tangent vector to the isoptic is always non-zero, and for $\beta \in\left(0, \frac{\pi}{2}\right)$ we have $\cos \beta>0$.

Along the positive $X$-axis the numerator of the curvature of the isoptic $C_{\alpha}$ is given by

$$
n_{\alpha}\left(-\frac{\alpha}{2}\right)=-\left(-a+6 \cos \frac{\alpha}{2}+2 \cos \frac{3 \alpha}{2}\right)\left(a-12 \cos \frac{\alpha}{2}+4 \cos \frac{3 \alpha}{2}\right) .
$$

Hence our problem simplifies to finding roots of functions

$$
f_{1}(a, \alpha)=-a+6 \cos \frac{\alpha}{2}+2 \cos \frac{3 \alpha}{2}
$$

and

$$
f_{2}(a, \alpha)=a-12 \cos \frac{\alpha}{2}+4 \cos \frac{3 \alpha}{2} .
$$

The denominator of the curvature of $C_{\alpha}$ at $t=-\frac{\alpha}{2}$ can be written as

$$
d_{\alpha}\left(-\frac{\alpha}{2}\right)=\frac{f_{1}(a, \alpha)^{3}}{\cos \frac{\alpha}{2}}
$$

so the function $f_{1}$ has no roots. Moreover, $f_{1}(a, \alpha)<0$ for $a>8$ and $\alpha \in(0, \pi)$ since it has no roots and $f_{1}(a, 0)<0$. For the function $f_{2}$ let us substitute $x=\cos \frac{\alpha}{2}$. Since $\alpha \in(0, \pi)$, we consider the resulting polynomial

$$
v_{2}(a, x)=16 x^{3}-24 x+a
$$

for $x \in(0,1]$ with the parameter $a>8$. To find the number of distinct roots of polynomial $v_{2}$ we apply the Sturm theorem (see Serret 1866; Sturm 1829) on the interval $(0,1]$. Constructing the Sturm sequence

$$
\begin{aligned}
& X_{0}(a, x)=v_{2}(a, x)=16 x^{3}-24 x+a \\
& X_{1}(a, x)=48 x^{2}-24 \\
& X_{2}(a, x)=16 x-a \\
& X_{3}(a, x)=-3 a x+24 \\
& X_{4}(a, x)=-\frac{128}{a}+a=\frac{(8 \sqrt{2}-a)(8 \sqrt{2}+a)}{a} \\
& X_{5}(a, x)=0
\end{aligned}
$$

the signs of their values for $a \in(8,8 \sqrt{2})$ are presented in Table 1 .

Hence for $a \in(8,8 \sqrt{2})$ we have $Z(0)-Z(1)=3-1=2$. Therefore, based on the Sturm theorem we conclude that the polynomial $v_{2}$ has two zeroes in the interval $(0,1]$ if the parameter $a \in(8,8 \sqrt{2})$. 
Table 1 The signs of values of the Sturm sequence of $v_{2}=16 x^{3}-24 x+a$ for $x \in(0,1]$ with the parameter $a \in(8,8 \sqrt{2})$

\begin{tabular}{llllllll}
\hline$i$ & 0 & 1 & 2 & 3 & 4 & 5 & \\
\hline sign of $X_{i}(a, 0)$ & + & - & - & + & - & 0 & $Z(0)=3$ \\
$\operatorname{sign}$ of $X_{i}(a, 1)$ & + & + & + & - & - & 0 & $Z(1)=1$ \\
\hline
\end{tabular}

Similar computations as those above yield that along the negative $X$-axis the curvature of isoptics of the given oval is always positive.

We conclude that if $a \in(8,8 \sqrt{2})$, then isoptics of the curve with support function $p(t)=a+\cos 3 t$ have two convexity limit angles. For example for $a=10$ those convexity limit angles are $\alpha_{1}=2 \arccos \left(\frac{\sqrt{21}-1}{4}\right)$ and $\alpha_{2}=\frac{2}{3} \pi$.

\section{Isoptics with three limit angles}

Theorem 2 Fix $15<a<17$ and let $C$ be the oval parametrized by the support function $p(t)=a+\cos 4 t$. For $\alpha \in(0, \pi)$, let $C_{\alpha}$ be the $\alpha$-isoptic of $C$. Then there exist angles $0<\alpha_{1}<\alpha_{2}<\alpha_{3}<\pi$ such that the point of $C_{\alpha}$ which intersects the coordinate axes has a positive curvature if $\alpha \in\left(0, \alpha_{1}\right) \cup\left(\alpha_{2}, \pi\right)$ and a negative curvature if $\alpha \in\left(\alpha_{1}, \alpha_{2}\right)$. Moreover, when $C_{\alpha}$ intersects the lines $y=x$ and $y=-x$, $C_{\alpha}$ has a positive curvature if $\alpha \in\left(0, \alpha_{3}\right)$ and a negative curvature if $\alpha \in\left(\alpha_{3}, \pi\right)$.

Proof We prove that the curve $C$ with support function $p(t)=a+\cos 4 t, a \in(15,17)$, is the example needed in our theorem.

We note that this curve has four axes of symmetry. Two of them, the $X$ and $Y$ axes, pass through the points of maximal curvature of $C$. The other two are the lines $y=x$ and $y=-x$, passing through the points of minimal curvature of $C$. Isoptics $C_{\alpha}$ are symmetric with respect to the same axes of symmetry as $C$. Moreover, the first derivative of the curvature of isoptics is zero along these symmetry axes. Similar to the development above for $p(t)=a+\cos 3 t$, we can show that orthogonal trajectories which start at $z\left(k \frac{\pi}{4}\right)$ are straight lines and lie on the axes of symmetry of the oval $C$. That is why we will study them along two orthogonal trajectories (see Fig. 1). The first of them starts at $z(0)$ and lies on the positive $X$-axis. It consists of the points $z\left(\frac{\pi}{4}\right)$ The second trajectory starts at $z\left(\frac{\pi}{4}\right)$ and lies on the line $y=x$. It is the set of the points $\left\{z_{\alpha}\left(\frac{\pi}{4}-\frac{\alpha}{2}\right), \alpha \in(0, \pi)\right\}$.

Along the positive $X$-axis the curvature of the isoptic $C_{\alpha}=\left\{z_{\alpha}(t), \quad t \in(0,2 \pi)\right\}$ is given by

$$
k_{\alpha}\left(-\frac{\alpha}{2}\right)=\frac{-\cos \frac{\alpha}{2}(4-a+8 \cos \alpha+3 \cos 2 \alpha)(-8+a-16 \cos \alpha+9 \cos 2 \alpha)}{(4-a+8 \cos \alpha+3 \cos 2 \alpha)^{3}} .
$$

Let us denote the following two functions:

$$
f_{1}(a, \alpha)=4-a+8 \cos \alpha+3 \cos 2 \alpha
$$


Table 2 The signs of the values of the Sturm sequence of the polynomial $v_{2}=18 x^{2}-16 x-17+a$ for $x \in(-1,1]$ with the parameter $a>15$

\begin{tabular}{llllll}
\hline$i$ & 0 & 1 & 2 & 3 & 4 \\
\hline sign of $X_{i}(a,-1)$ & + & - & + & 0 & $Z(-1)=2$ if $a<20$. \\
$\overline{5}$ & + & - & - & 0 & $Z(-1)=1$ if $a>20$. \\
$\overline{5}$ sign of $X_{i}(a, 1)$ & + & + & + & 0 & $Z(1)=0$ if $a<20$. \\
$\overline{5}$ & + & - & - & 0 & $Z(1)=1$ if $a>20$. \\
$\overline{5}$ & & & & & \\
\hline
\end{tabular}

and

$$
f_{2}(a, \alpha)=-8+a-16 \cos \alpha+9 \cos 2 \alpha .
$$

Since the curvature function of isoptics is well defined for $\alpha \in(0, \pi)$, the function $f_{1}$ has no roots. Hence

$$
k_{\alpha}\left(-\frac{\alpha}{2}\right)=0 \quad \Longleftrightarrow \quad f_{2}(a, \alpha)=0
$$

Let us substitute $x=\cos \alpha$. Since $\alpha \in(0, \pi)$ we consider the resulting polynomial

$$
v_{2}(a, x)=18 x^{2}-16 x-17+a
$$

for $x \in(-1,1]$ with the parameter $a>15$. Its Sturm sequence is

$$
\begin{aligned}
& X_{0}(a, x)=18 x^{2}-16 x-17+a, \\
& X_{1}(a, x)=36 x-16, \\
& X_{2}(a, x)=17+\frac{32}{9}-a, \\
& X_{3}(a, x)=0 .
\end{aligned}
$$

The signs of the values of the Sturm sequence are presented in Table 2.

Hence for $a \in(15,20 . \overline{5})$ we have $Z(-1)-Z(1)=2$ and using the Sturm theorem we conclude that the polynomial $v_{2}$ has two roots in the interval $(-1,1]$. If $a>20 . \overline{5}$ then $Z(-1)-Z(1)=0$ and we conclude that $v_{2}$ has no roots between -1 and 1 .

Now let us study the behaviour of the curvature of $C_{\alpha}$ along the orthogonal trajectory, starting at the point of $C$ with maximal curvature, for example, along the half-line $l(x)=z\left(\frac{\pi}{4}\right)+x \cdot(1,1)$, where $x>0$.

Along the half-line $l$ the curvature of the isoptic is given by

$$
k_{\alpha}\left(\frac{\pi}{4}-\frac{\alpha}{2}\right)=\frac{-\cos \frac{\alpha}{2}(4+a+8 \cos \alpha+3 \cos 2 \alpha)(-8-a-16 \cos \alpha+9 \cos 2 \alpha)}{(4+a+8 \cos \alpha+3 \cos 2 \alpha)^{3}} .
$$


Table 3 The signs of values of the Sturm sequence of $w_{2}=18 x^{2}-16 x-17-a$ for $x \in(-1,1]$ with the parameter $a>15$

\begin{tabular}{llllll}
\hline$i$ & 0 & 1 & 2 & 3 & \\
\hline $\operatorname{sign}$ of $X_{i}(a,-1)$ & + & - & + & 0 & $Z(-1)=2$ if $a<17$ \\
& - & - & + & 0 & $Z(-1)=1$ if $a>17$ \\
sign of $X_{i}(a, 1)$ & - & + & + & 0 & $Z(1)=1$
\end{tabular}

Similarly to the development above, we can consider the functions

$$
g_{1}(a, \alpha)=4+a+8 \cos \alpha+3 \cos 2 \alpha
$$

and

$$
g_{2}(a, \alpha)=-8-a-16 \cos \alpha+9 \cos 2 \alpha
$$

and substitute $x=\cos \alpha$. Then we can consider the polynomials

$$
w_{1}(a, x)=6 x^{2}+8 x+1+a
$$

and

$$
w_{2}(a, x)=18 x^{2}-16 x-17-a
$$

with the parameter $a>15$ for $x \in(-1,1]$. The function $g_{1}$ has no roots.

Let us construct the Sturm sequence of $w_{2}$

$$
\begin{aligned}
& X_{0}(a, x)=18 x^{2}-16 x-17-a, \\
& X_{1}(a, x)=36 x-16, \\
& X_{2}(a, x)=17+\frac{32}{9}+a, \\
& X_{3}(a, x)=0 .
\end{aligned}
$$

The signs of its values are presented in Table 3 .

Hence for $a \in(15,17)$ we have $Z(-1)-Z(1)=1$ and based on the Sturm theorem we conclude that the polynomial $w_{2}$ has one root in the interval $(-1,1]$. If $a>17$ then $Z(-1)-Z(1)=0$ which implies that $w_{2}$ has no roots between -1 and 1 .

Combining the results of the two trajectories we conclude that isoptics of the oval parametrized by the support function $p(t)=a+\cos 4 t$ have three convexity limit 
angles if $a \in(15,17)$. We can describe them by the functions

$$
\begin{aligned}
& \alpha_{1}(a)=\arccos \frac{8+\sqrt{2} \sqrt{185-9 a}}{18}, \\
& \alpha_{2}(a)=\arccos \frac{8-\sqrt{2} \sqrt{185-9 a}}{18} \\
& \alpha_{3}(a)=\arccos \frac{8-\sqrt{2} \sqrt{185+9 a}}{18}
\end{aligned}
$$

of the variable $a$.

We note that for each $a \in(15,17)$ we have $\alpha_{1}(a)<\alpha_{2}(a)<\alpha_{3}(a)$. So for $\alpha \in\left(0, \alpha_{1}(a)\right) \cup\left(\alpha_{2}(a), \alpha_{3}(a)\right)$, the isoptics of the oval $C$ are convex and for $\alpha \in$ $\left(\alpha_{1}(a), \alpha_{2}(a)\right) \cup\left(\alpha_{3}(a), \pi\right)$ they are nonconvex.

\section{General case for $p(t)=a+\cos n t$}

For $a>n^{2}-1$ the curve $C$ parametrized by the support function $p(t)=a+\cos n t$ is an oval and has $n$ axes of symmetry. Isoptics of such ovals also have $n$ symmetry axes, passing through the points of extremal curvature of $C$ and coinciding with the orthogonal trajectories of the evolutions of the ovals. Those isoptics are invariant with respect to rotations about the origin by the angles $\frac{2 k \pi}{n}$, where $k=1, \ldots, n$. That is why we consider the curvature of the isoptics of the oval with the support function $p(t)=a+\cos n t$, where $a>n^{2}-1$ along two half-lines.

- The first half-line starts at $z(0)$ and lies on the positive $X$-axis. It starts on the given oval, at the point in which it has maximal curvature. It is a set of the points $\left\{z_{\alpha}\left(-\frac{\alpha}{2}\right), \alpha \in(0, \pi)\right\}$ and we denote it $\mathbf{I}$.

- The second half-line starts at $z\left(\frac{\pi}{n}\right)$ and passes though the points $z_{\alpha}\left(\frac{\pi}{n}-\frac{\alpha}{2}\right)$ for $\alpha \in(0, \pi)$. It starts on the given oval, at the point in which it has minimal curvature. We denote it II.

For the curvature

$$
k_{\alpha}(t)=\frac{\left[z_{\alpha}^{\prime}(t), z_{\alpha}^{\prime \prime}(t)\right]}{\left|z_{\alpha}^{\prime}(t)\right|^{3}}
$$

let us denote

$$
n_{\alpha}(t)=\left[z_{\alpha}^{\prime}(t), z_{\alpha}^{\prime \prime}(t)\right] \sin ^{2} \alpha, \quad d_{\alpha}(t)=\left|z_{\alpha}^{\prime}(t)\right|^{3} \sin ^{2} \alpha
$$

Along the trajectory $\mathbf{I}$ we obtain

$$
d_{\alpha}\left(-\frac{\alpha}{2}\right)=\frac{1}{\sin \alpha}\left(\left(a+\cos \frac{n s}{2}\right) \sin \frac{\alpha}{2}-n \cos \frac{\alpha}{2} \sin \frac{n \alpha}{2}\right)^{3}
$$


and

$$
n_{\alpha}\left(-\frac{\alpha}{2}\right)=-f_{1}(\alpha, a, n) \cdot f_{2}(\alpha, a, n)
$$

where

$$
f_{1}(\alpha, a, n)=-2 a \sin \frac{\alpha}{2}+(n+1) \sin \frac{(n-1) \alpha}{2}+(n-1) \sin \frac{(n+1) \alpha}{2}
$$

and

$$
f_{2}(\alpha, a, n)=2 a \sin \frac{\alpha}{2}-(n+1)^{2} \sin \frac{(n-1) \alpha}{2}+(n-1)^{2} \sin \frac{(n+1) \alpha}{2} .
$$

We need to calculate the number of roots of the functions $f_{1}(\alpha, a, n)$ and $f_{2}(\alpha, a, n)$. The functions vary with $\alpha$, and have parameters $a$ and $n$. From the form of the denominator we observe that $f_{1}$ has no roots. We can also check it applying the same procedure for $f_{1}$ as we will use for $f_{2}$, below. We perform a change of variables in $f_{2}$, in order to obtain a polynomial of a real variable. We will do this for two cases: $n$ odd and $n$ even.

For $n$ odd let us substitute $u=\frac{\alpha}{2}$ where $u \in\left(0, \frac{\pi}{2}\right)$. Then

$$
f_{2}(u, a, n)=2 a \sin u-(n+1)^{2} \sin (n-1) u+(n-1)^{2} \sin (n+1) u .
$$

Using the trigonometric identity for $\sin n x$ we get

$$
\begin{aligned}
f_{2}(u, a, n)= & \sin u\left(2 a-(n+1)^{2} \sum_{i=0}^{\left\lfloor\frac{n-1}{2}\right\rfloor}(-1)^{i} \cdot\left(\begin{array}{c}
n-1 \\
2 i+1
\end{array}\right) \cos ^{n-2 i-2} u \sin ^{2 i} u\right. \\
& \left.+(n-1)^{2} \sum_{i=0}^{\left\lfloor\frac{n+1}{2}\right\rfloor}(-1)^{i} \cdot\left(\begin{array}{c}
n+1 \\
2 i+1
\end{array}\right) \cos ^{n-2 i} u \sin ^{2 i} u\right) .
\end{aligned}
$$

Since $\sin u>0$ for $u \in\left(0, \frac{\pi}{2}\right)$, using the Pythagorean trigonometric identity and the substitution $x=\cos u$, with $x \in(0,1)$, for the function $\frac{f_{2}}{\sin u}$ we obtain the polynomial

$$
\begin{aligned}
v_{2}(x, a, n)= & 2 a-(n+1)^{2} \sum_{i=0}^{\left\lfloor\frac{n-1}{2}\right\rfloor}(-1)^{i} \cdot\left(\begin{array}{c}
n-1 \\
2 i+1
\end{array}\right) x^{n-2 i-2}\left(1-x^{2}\right)^{i} \\
& +(n-1)^{2} \sum_{i=0}^{\left\lfloor\frac{n+1}{2}\right\rfloor}(-1)^{i} \cdot\left(\begin{array}{c}
n+1 \\
2 i+1
\end{array}\right) x^{n-2 i}\left(1-x^{2}\right)^{i} .
\end{aligned}
$$

We now need to calculate the number of zeroes for $x \in(0,1)$. 
We can repeat the above algorithm for even $n$. However, if we do it in a slightly different way we will obtain polynomials of lower degrees. Noticing that $n=2 k$ for $k \in \mathbb{Z}$, let us consider the function

$$
\begin{aligned}
f_{2}(\alpha, a, k)= & 2 \sin \frac{\alpha}{2}\left(a+\left(4 k^{2}+1\right) \cos k \alpha\right. \\
& \left.-4 k(\cos \alpha+1) \sum_{i=0}^{\left\lfloor\frac{k}{2}\right\rfloor}(-1)^{i} \cdot\left(\begin{array}{c}
k \\
2 i+1
\end{array}\right) \cos ^{k-2 i-1} \alpha \sin ^{2 i} \alpha\right) .
\end{aligned}
$$

Substituting $x=\cos \alpha$ where $x \in(-1,1)$ and using the trigonometric identity for $\cos n x$, the function $\frac{f_{2}}{2 \sin \frac{\alpha}{2}}$ becomes the polynomial

$$
\begin{aligned}
v_{2}(x, a, n, k)= & a+\left(4 k^{2}+1\right) \sum_{i=0}^{\left\lfloor\frac{k}{2}\right\rfloor}(-1)^{i} \cdot\left(\begin{array}{c}
k \\
2 i
\end{array}\right) x^{k-2 i}\left(1-x^{2}\right)^{i} \\
& -4 k(x+1) \sum_{i=0}^{\left\lfloor\frac{k}{2}\right\rfloor}(-1)^{i} \cdot\left(\begin{array}{c}
k \\
2 i+1
\end{array}\right) x^{k-2 i-1}\left(1-x^{2}\right)^{i}
\end{aligned}
$$

Our approach would be similar to the previous cases, applying the Sturm theorem for finding the number of roots. However, since the polynomial $v_{2}(x, a, n, k)$ depends on the degree $n$, we need to generalize this theorem.

Along the trajectory II, after similar computations we get the following formulae

$$
n_{\alpha}\left(\frac{\pi}{n}-\frac{\alpha}{2}\right)=-g_{1}(\alpha, a, n) \cdot g_{2}(\alpha, a, n)
$$

and

$$
d_{\alpha}\left(\frac{\pi}{n}-\frac{\alpha}{2}\right)=\frac{\left(g_{1}(\alpha, a, n)\right)^{3}}{\sin \alpha}
$$

where

$$
g_{1}(\alpha, a, n)=2 a \sin \frac{\alpha}{2}+(n+1) \sin \frac{(n-1) \alpha}{2}+(n-1) \sin \frac{(n+1) \alpha}{2}
$$

and

$$
g_{2}(\alpha, a, n)=-2 a \sin \frac{\alpha}{2}-(n+1)^{2} \sin \frac{(n-1) \alpha}{2}+(n-1)^{2} \sin \frac{(n+1) \alpha}{2}
$$

The function $g_{1}(\alpha, a, n)$ has no roots. We want to transform the function $g_{2}(\alpha, a, n)$ into a real variable polynomial. For $n$ odd using the substitution $x=\cos \frac{\alpha}{2}$ we obtain 
the following polynomial

$$
\begin{aligned}
w_{2}(x, a, n)= & -2 a-(n+1)^{2} \sum_{i=0}^{\left\lfloor\frac{n-1}{2}\right\rfloor}(-1)^{i} \cdot\left(\begin{array}{c}
n-1 \\
2 i+1
\end{array}\right) x^{n-2 i-2}\left(1-x^{2}\right)^{i} \\
& +(n-1)^{2} \sum_{i=0}^{\left\lfloor\frac{n+1}{2}\right\rfloor}(-1)^{i} \cdot\left(\begin{array}{c}
n+1 \\
2 i+1
\end{array}\right) x^{n-2 i}\left(1-x^{2}\right)^{i},
\end{aligned}
$$

for the interval $(0,1)$. For $n$ even substituting $x=\cos u$ we obtain

$$
\begin{aligned}
w_{2}(x, a, n, k)= & a+\left(4 k^{2}+1\right) \sum_{i=0}^{\left\lfloor\frac{k}{2}\right\rfloor}(-1)^{i} \cdot\left(\begin{array}{c}
k \\
2 i
\end{array}\right) x^{n-2 i}\left(1-x^{2}\right)^{i} \\
& -4 k(x+1) \sum_{i=0}^{\left\lfloor\frac{k}{2}\right\rfloor}(-1)^{i} \cdot\left(\begin{array}{c}
k \\
2 i+1
\end{array}\right) x^{k-2 i-1}\left(1-x^{2}\right)^{i},
\end{aligned}
$$

where $n=2 k$ and for which we are looking for the number of roots in the interval $(-1,1)$. This number of zeroes depends on the parameter $a$ and we need to find such $a_{1}(n)$, that for $a \in\left(n^{2}-1, a_{1}(n)\right)$ the polynomials $v_{2}$ have the largest possible number of zeros in the appropriate intervals.

Whereas results for arbitrary $n$ are as yet unknown, those for some fixed values of $n$ are. We also put forward a hypothesis concerning the general case.

For $n=5$ the polynomial

$$
v_{2}(x, a, 5)=a+256 x^{5}-400 x^{3}+120 x
$$

has two roots for $a \in\left(24, \sqrt{\frac{6975}{8}+\frac{129^{3 / 2}}{8}}\right) \approx(24,32.48)$ and the polynomial

$$
w_{2}(x, a, 5)=-a+256 x^{5}-400 x^{3}+120 x
$$

has two roots for $a \in\left(24, \frac{1}{2} \sqrt{\frac{3}{2}(2325-43 \sqrt{129})}\right) \approx(24,26.24)$. Hence we have four convexity limit angles if $a \in\left(24, \frac{1}{2} \sqrt{\frac{3}{2}(2325-43 \sqrt{129})}\right) \approx(24,26.24)$. Those limit angles satisfy the inequality $0<\alpha_{1}<\alpha_{2}<\frac{\pi}{2}<\alpha_{3}<\alpha_{4}<\pi$. Moreover, $\alpha_{1}$ and $\alpha_{2}$ lie on trajectory I and $\alpha_{3}$ and $\alpha_{4}$ lie on trajectory II.

For $n=6$ the polynomial

$$
v_{2}(x, a, 6,3)=a+100 x^{3}-48 x^{2}-99 x+12
$$

has three roots for $a \in(35,37)$ and the polynomial

$$
w_{2}(x, a, 6,3)=-a+100 x^{3}-48 x^{2}-99 x+12
$$


Table 4 The maximal number of convexity limit angles along orthogonal trajectories I and II

\begin{tabular}{llllllllllllllllll}
\hline$n$ & 2 & 3 & 4 & 5 & 6 & 7 & 8 & 9 & 10 & 11 & 12 & 13 & 14 & 15 & 16 & 17 & 18 \\
\hline I & 1 & 2 & 2 & 2 & 3 & 4 & 4 & 4 & 5 & 6 & 6 & 6 & 7 & 8 & 8 & 8 & 9 \\
II & 0 & 0 & 1 & 2 & 2 & 2 & 3 & 4 & 4 & 4 & 5 & 6 & 6 & 6 & 7 & 8 & 8 \\
\hline
\end{tabular}

has two roots for $a \in\left(35, \frac{7}{625}(127 \sqrt{889}-416)\right) \approx(35,37.75)$. Hence we have five convexity limit angles if $a \in(35,37)$. Those limit angles satisfy the inequality $0<\alpha_{1}<\alpha_{2}<\frac{\pi}{2}<\alpha_{3}<\alpha_{4}<\frac{5}{6} \pi<\alpha_{5}<\pi$. Moreover, $\alpha_{1}, \alpha_{2}$ and $\alpha_{5}$ lie on trajectory I and $\alpha_{3}$ and $\alpha_{4}$ lie on trajectory II.

The maximal number of convexity limit angles along the orthogonal trajectories starting at the points of $C$ of the largest (I) and the smallest (III) curvature, are obtained from computer experimentation, and are presented in Table 4.

These observations led us to formulate the following hypothesis

Hypothesis For the isoptics of the curve with support function $p(t)=a+\cos n t$, $a>n^{2}-1$, for $a \in\left(n^{2}-1, a_{1}(n)\right)$, where $a_{1}(n)>n^{2}-1$, there exist $n-1$ convexity limit angles. These limit angles can be divided into two groups of sizes $v(n)$ and $w(n)$, where $v(n)+w(n)=n-1$. The first group of size $v(n)$ appears on the orthogonal trajectory, starting at the point $z\left(\frac{2 k \pi}{n}\right)$. The second group of size $w(n)$ is found on the orthogonal trajectory, starting at the point $z\left(\frac{\pi}{n}+\frac{2 k \pi}{n}\right)$, where $k=0,1, \ldots, n-1$,

$$
\begin{gathered}
v(n)= \begin{cases}2 k, & n=4 k+0 \\
2 k, & n=4 k+1 \\
2 k+1, & n=4 k+2 \\
2(k+1), & n=4 k+3\end{cases} \\
w(n)= \begin{cases}2 k-1, & n=4 k+0 \\
2 k, & n=4 k+1 \\
2 k, & n=4 k+2 \\
2 k, & n=4 k+3\end{cases}
\end{gathered}
$$

and $v(n)+w(n)=n-1$.

Open Access This article is licensed under a Creative Commons Attribution 4.0 International License, which permits use, sharing, adaptation, distribution and reproduction in any medium or format, as long as you give appropriate credit to the original author(s) and the source, provide a link to the Creative Commons licence, and indicate if changes were made. The images or other third party material in this article are included in the article's Creative Commons licence, unless indicated otherwise in a credit line to the material. If material is not included in the article's Creative Commons licence and your intended use is not permitted by statutory regulation or exceeds the permitted use, you will need to obtain permission directly from the copyright holder. To view a copy of this licence, visit http://creativecommons.org/licenses/by/4.0/. 


\section{References}

Cieślak, W., Miernowski, A., Mozgawa, W.: Isoptics of a closed strictly convex curve, Global differential geometry and global analysis (Berlin, 1990). Lect. Notes Math. 1481, 28-35 (1991)

Dana-Picard, T.: An automated study of isoptic curves of an astroid. J. Symb. Comput. 97, 56-68 (2020)

Kunkli, R., Papp, I., Hoffmann, M.: Isoptics of Bézier curves. Comput. Aided Geom. Des. 30, $78-84$ (2013)

Lawrence, D.: A Catalog of Special Plane Curves. Dover Publications, New York (1972)

Miernowski, A., Mozgawa, W.: On some geometric condition for convexity of isoptics. Rend. Sem. Mat. Univ. Politec. Torino 55(2), 93-98 (1997)

Serret, J. A.: Cours d'Algébre Supérieure, p. 227, Bachelier, Paris, 1849, Second ed. Mallet-Bachelier, Paris, 1854, Third ed. Gauthier-Villars, Paris (1866)

Skrzypiec, M.: Dual curves to isoptics of ovals. Bull. Soc. Sci. Lett. Łódź, Sér. Rech. Déform. 68(1):85-96 (2018). https://doi.org/10.26485/0459-6854/2018/68.1/6

Sturm, Ch.: Analyse d'un mémoire sur la résolution des équations numeriques. Bulletin de Férussac 11, 419-422 (1829)

Weiss, G., Martini, H.: On curves and surfaces in illumination geometry. J. Geom. Graphics 4, 169-180 (2000)

Wunderlich, W.: Contributions to the geometry of cam mechanisms with oscillating followers. J. Mech. 6(1), 1-20 (1971)

Publisher's Note Springer Nature remains neutral with regard to jurisdictional claims in published maps and institutional affiliations. 